\title{
Dependence of the Crossover Exponent with the Diffusion Rate in the Generalized Contact Process Model
}

\author{
W. G. Dantas* and M. J. de Oliveira ${ }^{\dagger}$ \\ Universidade de São Paulo, Caixa Postal 66318 05315-970 São Paulo, SP, Brazil \\ J. F. Stilck \\ Instituto de Física, Universidade Federal Fluminense, 24210-340, Niterói, RJ, Brazil
}

Received on 17 December, 2007

\begin{abstract}
We study how the crossover exponent, $\phi$, between the directed percolation (DP) and compact directed percolation (CDP) behaves as a function of the diffusion rate in a model that generalizes the contact process. Our conclusions are based in results pointed by perturbative series expansions and numerical simulations, and are consistent with a value $\phi=2$ for finite diffusion rates and $\phi=1$ in the limit of infinite diffusion rate.
\end{abstract}

Keywords: Phase transitions; Absorbing states; Crossover exponents; Diffusion process

\section{INTRODUCTION}

The research of the critical properties of nonequilibrium systems that undergo a phase transition between an active and an absorbing phase has been increasing in the last decade $[1,2]$. Although many aspects of these transitions already have been well established in the literature [3], the absence of a general theory for such systems leads to many open questions. These questions, in general, only can be answered partially and these answers are obtained, among other approaches through numerical simulations, a very useful tool in this task [4], and perturbative series expansions [5, 6], a powerful technique that, in some situations, may lead to good numerical precision of the estimates for critical properties.

One main challenge in this field is determine, as precise is possible, a separation scheme of the models with the same critical properties or, using other words, determine the universality classes for these phase transitions [7]. Although we do not have a systematic way to accomplish this task, one conclusion that seems to be correct is that all systems with a scalar order parameter, local interactions and without conservation laws belong to the same universality class called as Directed Percolation (DP) class. This statement is known as DP conjecture [8]. The vast majority of the models which exhibit a phase transition between an active and absorbing state belong to this class, indicating that it is very robust. Thus, it is important to study different models and determine their universality class and it is essential to use approaches that permit us to estimate, as precisely as possible, the critical exponents for each model.

In a recent paper, we estimated the crossover exponent between the DP and DCP classes [9]. Using the perturbative supercritical series expansion formalism, proposed by Dickman and Jensen [5], we obtain a very precise estimates for this exponent in a generalized contact process (CP) model,

\footnotetext{
*Electronic address: wgd@gibbs.if.usp.br

†Electronic address: oliveira@if.usp.br

Electronic address: jstilck@if.uff.br
}

which lead us to conjecture that $\phi=2$, in accordance with upper bound estimates [10] and simulational results for the Domany-Kinzel cellular automaton [11], which may indicate that this exponent is the same for similar models with paralell and sequencial updates. However, in an extended version of the generalized model (including a diffusion process) we were not be able to determine the behavior of the crossover exponent $\phi$ as a function of the diffusion rate in the limit of high diffusion rate [12]. Our results show only that, in a region of weak diffusion, the crossover exponent still is $\phi=2$. In this work, using numerical simulations to estimate this exponent we expect answer the question if the crossover between the DP and CDP classes changes with the diffusion rate.

This work is organized as follows. In section II, we present the model and discuss mean-field and perturbative series results of the reference [12]. In section III we describe the numerical simulational scheme and show the new results that we obtained through it. Finally, conclusions and perspectives may be found in section IV.

\section{MODEL}

In a one-dimensional lattice with periodic boundary conditions each site is empty or occupied by one particle. Thus, a configuration of the system at time $t$ is given by the vector $|\eta\rangle=\left|\eta_{1}, \eta_{2}, \ldots, \eta_{N}\right\rangle$, where $\eta_{i}=0,1$ if the site is empty or occupied, respectively. The markovian evolution of the system is given by the following rules:

- We choose, randomly, a site $i$.

- If this site is empty, then it becomes occupied with a transition rate equal to $p_{a} n / 2$, where $n$ is the number of the particles in the first neighbor sites of $i$.

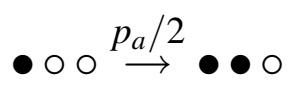


- If the site $i$ is occupied, it may become empty by two processes:

1. Spontaneously, independently of its neighborhood, with rate $p_{c}$

$$
\stackrel{p_{c}}{\rightarrow} \text { ○ }
$$

2. Through contact with first neighbor empty sites with a rate $p_{b}(2-n) / 2$.

$$
\circ \bullet \circ \stackrel{p_{b}}{\longrightarrow} \circ \circ \circ
$$

- Finally, we have a diffusive process for the particles, occurring with a rate $\tilde{D}$.

$$
\bullet \stackrel{\tilde{D}}{\rightarrow} \circ
$$

We define the parameters in such way that $p_{a}+p_{b}+p_{c}=1$ and $0<\tilde{D}<\infty$. For convenience, we will discuss our results in the space $\left(p_{a}, p_{c}, \tilde{D}\right)$. This model reduces to the CP model with diffusion if we put $p_{b}=0$ and to the voter model or linear Glauber model at zero temperature for $p_{a}=p_{b}$ and $p_{c}=0$. In the first case we have exponents that belongs to the DP universality class and in the last case the phase transition belongs to the CDP universality class. Thus, in the neighborhood of $p_{c}=0$, we have a crossover between these classes.

For a certain fixed value of the diffusion rate, the behavior of the any stationary density close to the multicritical point, should be given by the scaling form,

$$
g\left(\Delta p_{a}, \Delta p_{c}, \tilde{D}\right) \sim \Delta p_{a}^{e_{g}(\tilde{D})} F\left(\frac{\Delta p_{c}}{\left|\Delta p_{a}\right|^{\phi(\tilde{D})}}\right),
$$

where $\Delta p_{a}=p_{a}-1 / 2, \Delta p_{c}=p_{c}, e_{g}(\tilde{D})$ is a critical exponent related with the density $g$ and $\phi(\tilde{D})$ is the crossover exponent. The scaling function $F(z)$ is singular at a value $z_{0}(\tilde{D})$ of its argument, which corresponds to the critical line for a certain diffusion rate value, $\tilde{D}$. Using the scaling form (1), we obtain that the critical line is asymptotically given by $p_{c}=z_{0}(\tilde{D}) \Delta p_{a}^{\phi(\tilde{D})}$.

In a recent study [12], we have derived solutions for the cluster dynamics approximation in the one-, two- and threesite level. In the first case (simple mean-field approximation) the critical line is $p_{a}=1 / 2$, independently of the diffusion rate. Thus, the crossover exponent in this approximation is $\phi=0$. In the two-site level, we have a solution that shows three values of this exponent: $\phi=2$, if $\tilde{D}=0, \phi=1$, if $0<$ $\tilde{D}<\infty$ and $\phi=0$ for the infinite diffusion rate. Finally, in the three-site level of mean-field approximation, we obtain only a numerical estimate of the critical line. In this calculation, at sufficiently small diffusion rates, the crossover exponent value is $\phi \approx 2$ but, in an intermediate region of the diffusion rate, a slight deviation to smaller values appears, converging to $\phi \approx 1$ at high diffusion rates. It is probable that, in meanfield approximations of higher order, the interval of values of $\tilde{D}$ for which $\phi \approx 2$ grows.

On the other hand, in the same work, a perturbative series approach furnishes $\phi=2$, at least up to $\tilde{D} \approx 0.3$. Unfortunately, we were not able to determine the crossover exponent values for larger diffusion rates. Therefore, it would be interesting to use a numerical simulational approach to investigate how the crossover exponent behaves as a function of the diffusion rate, paricularly for large values of this rate.

\section{NUMERICAL SIMULATIONS}

In order to reach higher values of the diffusion rate and determine the behavior of the crossover exponent we use timedependent numerical simulations for the model. These simulations are carried out using a single seed in a lattice with $N=10000$ sites and periodic boundary conditions to avoid finite-size effects. The simulation is done for discrete time, and may be described by the following steps:

1. A list of all sites occupied by particles is stored, and at each time step one of them is chosen randomly.

2. Once the site is chosen, a random number $\mathrm{p}$ uniformly distributed in the interval $[0,1]$ is generated, if $p<$ $D=\tilde{D} /(1+\tilde{D})$ a pair hole-particle of first neighbors is switched, if possible. Otherwise, we choose a reaction: creation or annihilation. With a probability $p_{a}$ an empty site, in one of the first neighbors, is occupied, if possible. Otherwise, a particle at the chosen site will be annihilated either through the spontaneous or through the autocatalytic process.

3. To define the process switching a particle to a hole, another random number $q$ is generated. If $q<p c /(1-p a)$ the change is spontaneous, otherwise it will happen with a probability proportional to the number of empty sites in first neighbors of the chosen site.

4. The time interval associated with the steps above is $d t=$ $1 / N_{A}$, where $N_{A}$ is the number of the sites occupied by particles before the step. The process is repeated until either a maximum number of steps $n_{\max }$ is attained or the absorbing state $N_{A}=0$ is reached.

5. Several runs are done and mean values are calculated as functions of time.

Typically, we use in simulations, $n_{\max }=10^{5}$ steps and 2000 runs. We estimated the critical line for some values of the diffusion rate $D$ as can be seen in the Fig. (1). 

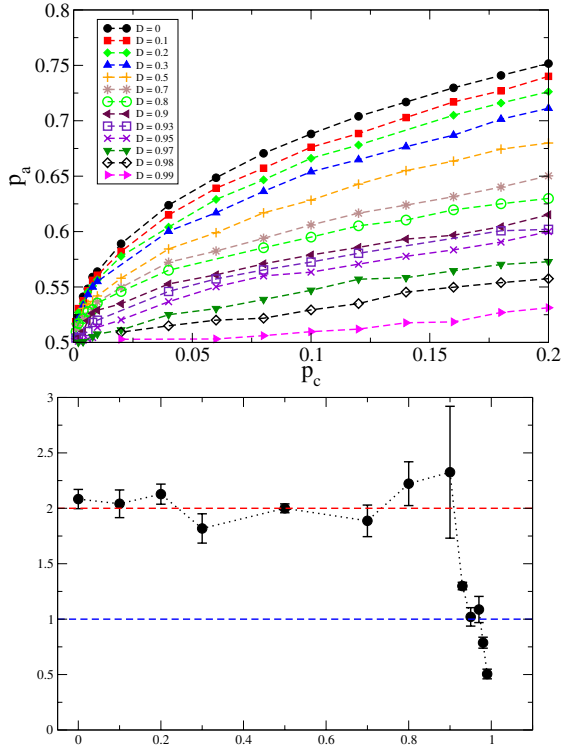

FIG. 1: Critical line and crossover exponents estimated in the threesite level mean-field approach (for $D=\tilde{D} /(1+\tilde{D})$ fixed). At small diffusion rate, the crossover exponent is $\phi \approx 2$.
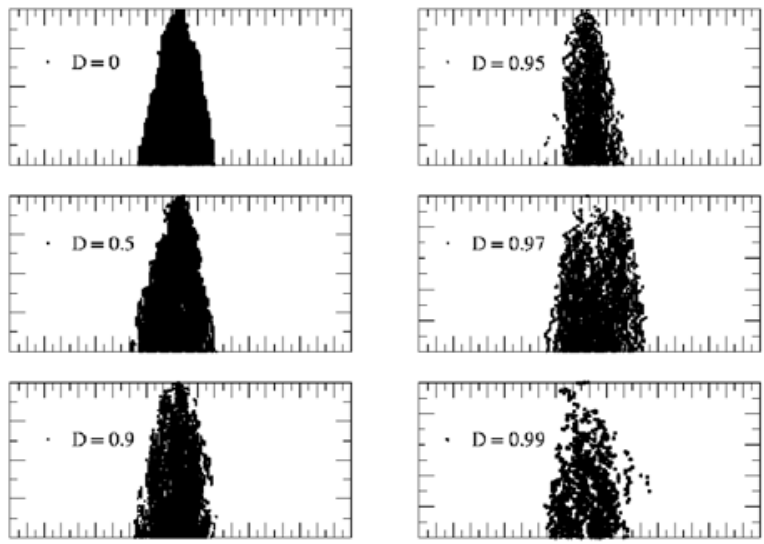

FIG. 2: Spreading of particles with a single seed as initial condition. The time arrow points down and this behavior is obtained for $p_{c}=$ 0.02 and $p_{a}>p_{a}^{c}$.

Our results suggest that the crossover exponent is $\phi=2$ up to $D \approx 0.9$, with decreasing estimates at higher diffusion rates. Although, we believe that this behavior is a numerical artifact due to an imprecision estimate of the critical points at this region, indicating a trend to vanishing $\phi$ in the infinite diffusion rate limit. Therefore, our results seems indicate that the crossover exponent between the DP and CDP universality classes in the generalized contact process is

$$
\phi= \begin{cases}0 & \text { if } \mathrm{D}=1 \\ 2 & \text { otherwise. }\end{cases}
$$

The change in the crossover exponent value seems be related to the disapearance of compact clusters of particles. The compact clusters are a signature of the CDP behavior. In the Fig. (2) we show that these clusters are no longer present when the diffusion rate is large.

We do not have a rigorous argument to relate the loss of the compact cluster to the change of the crossover exponent. It would be interesting study in a similar model if this apparent connection is correct.

\section{CONCLUSIONS}

In this work we study how the diffusion rate affects the crossover exponent between the DP and CDP universality classes. In a preliminar work [12], using perturbative series approach, we determine the value $\phi \approx 2$ up to $D \approx 0.3$ and due to limitations in the series derivation, we were not able to reach larger values for the diffusion rate.

However, the numerical simulation approach allows us to obtain estimates for the crossover exponent in a region closer to the limit where the diffusion rate diverges. Our results suggested that the crossover exponent is $\phi=2$ for all finite diffusion rates and $\phi=0$ at infinte diffusion rate, recovering the simple mean-field result, as expected. Besides, the change of the crossover exponent value in the infinite diffusion rate limit could be related to the loss of compact clusters of particles, although this cannot be proven by our results, but only suggested.

In a future work, we intend to realize quasi-stationary simulations, according the Oliveira and Dickman scheme [14], trying to obtain better results for the region with $D>0.9$.

\section{Acknowledgement}

W.G. Dantas acknowledges the financial support from Fundação de Amparo à Pesquisa do Estado de São Paulo (FAPESP) under Grant No. 05/04459-1 and JFS acknowledges funding by project PRONEX-CNPq-FAPERJ/171.1682003.
[1] J. Marro and R. Dickman, Nonequilibrium Phase Transitions in Lattice Models (Cambridge University Press, Cambridge, 1999)

[2] K. A. Takeuchi, M. Kuroda, H. Chaté and M. Sano, condmat/0706.4151 (2007).
[3] H. Hinrichsen, Adv. Phys.,49, 815 (2000).

[4] P. Grassberger and A. De La Torre, Ann.Phys. 122, 373 (1979).

[5] I. Jensen and R. Dickman, J. Stat. Phys. 71, 89 (1993).

[6] M.J. de Oliveira, J. Phys. A 39, 11131 (2006).

[7] G. Odor, Rev. Mod. Phys. 76, 663 (2004). 
[8] H. K. Janssen, Z. Phys. B, 42, 151 (1981); P. Grassberger, Z. Phys. B, 47, 365 (1982).

[9] W.G. Dantas and J.F. Stilck, J. Phys. A, 38, 5841 (2005.)

[10] T. M. Liggett, Ann. Appl. Probab. xx, yy (1994).

[11] A. Yu. Tretyakov, N. Inui, M. Katori, and H. Tsukahara, condmat/9509061 (1995).
[12] W.G. Dantas and J.F. Stilck, J. Stat. Mech., 06, P06019 (2007.)

[13] M. E. Fisher and R. M. Kerr, Phys. Rev. Lett. 32, 667 (1977).

[14] M.M. de Oliveira and R. Dickman, Phys. Rev. E, 71, 016129 (2005.) 